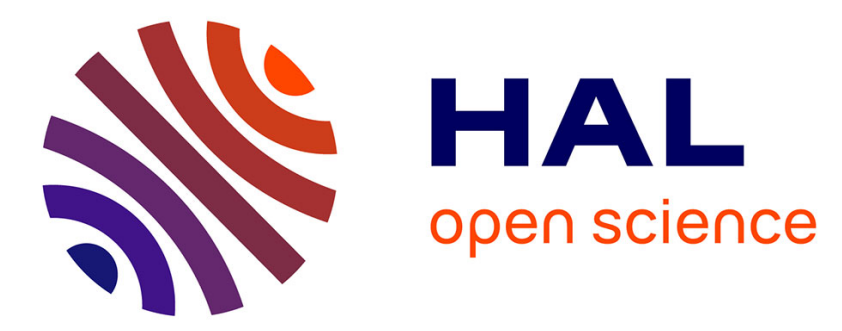

\title{
Investigation of interfacial stiffnesses of a tri-layer using Zero-Group Velocity Lamb modes
}

Sylvain Mezil, Francois Bruno, Samuel Raetz, Jérôme Laurent, Daniel Royer, Claire Prada

\section{- To cite this version:}

Sylvain Mezil, Francois Bruno, Samuel Raetz, Jérôme Laurent, Daniel Royer, et al.. Investigation of interfacial stiffnesses of a tri-layer using Zero-Group Velocity Lamb modes. Journal of the Acoustical Society of America, 2015, 138 (5), pp.3202 - 3209. 10.1121/1.4934958 . hal-01889422

\section{HAL Id: hal-01889422}

https://hal-univ-lemans.archives-ouvertes.fr/hal-01889422

Submitted on 9 Oct 2018

HAL is a multi-disciplinary open access archive for the deposit and dissemination of scientific research documents, whether they are published or not. The documents may come from teaching and research institutions in France or abroad, or from public or private research centers.
L'archive ouverte pluridisciplinaire HAL, est destinée au dépôt et à la diffusion de documents scientifiques de niveau recherche, publiés ou non, émanant des établissements d'enseignement et de recherche français ou étrangers, des laboratoires publics ou privés. 


\title{
Investigation of interfacial stiffnesses of a tri-layer using Zero-Group Velocity Lamb modes
}

\author{
Sylvain Mezil, Francois Bruno, Samuel Raetz, Jérôme Laurent, Daniel Royer, \\ and Claire Prada ${ }^{a)}$ \\ ESPCI Paris Tech, PSL Research University, CNRS, Institut Langevin, 1 rue Jussieu, F-75005, Paris, France
}

(Received 18 June 2015; revised 29 September 2015; accepted 18 October 2015; published online 20 November 2015)

Zero-Group Velocity (ZGV) Lamb waves are studied in a structure composed of two plates bonded by an adhesive layer. The dispersion curves are calculated for a Duralumin/epoxy/Duralumin sample, where the adhesion is modeled by a normal and a tangential spring at both interfaces. Several ZGV modes are identified and their frequency dependence on interfacial stiffnesses and on the bonding layer thickness is numerically studied. Then, experiments achieved with laser ultrasonic techniques are presented. Local resonances are measured using a superimposed source and probe. Knowing the thicknesses and elastic constants of the Duralumin and epoxy layers, the comparison between theoretical and experimental ZGV resonances leads to an evaluation of the interfacial stiffnesses. A good agreement with theoretical dispersion curves confirms the identification of the resonances and the parameter estimations. This non-contact technique is promising for the local evaluation of bonded structures. (C) 2015 Acoustical Society of America.

[http://dx.doi.org/10.1121/1.4934958]

$[\mathrm{ANN}]$

Pages: $3202-3209$

\section{INTRODUCTION}

The characterization of the elastic coupling of two plates by an adhesive layer is of primary importance in the evaluation of bonded structures. Various acoustic methods have been proposed to measure adhesion using reflection ${ }^{1}$ or transmission ${ }^{2}$ of bulk acoustic waves. These methods are more suitable for disbond detection than for adhesion characterization. Other techniques exploit guided waves, such as Lamb modes or shear horizontal modes, propagating along the structure. ${ }^{3-7}$ However, these non-local methods are not very sensitive and provide an average estimation of the bonding quality. A thorough review on ultrasound nondestructive testing of adhesive bond was conducted by Castaings. ${ }^{8}$

An alternative approach using Zero-Group Velocity (ZGV) Lamb modes has been the object of recent studies. The existence of backward waves, and consequently of ZGV modes, was studied theoretically for solid/liquid/solid structures. ${ }^{9-11}$ Laser ultrasonic experiments confirmed the narrow resonances observed at $\mathrm{ZGV}$ frequencies in the case of a thin fluid layer between two solid plates. ${ }^{11}$ It was also shown experimentally that ZGV modes exist in plates bonded by a solid layer and are promising to evaluate the bonding quality. ${ }^{12-14}$ High sensitivity and local measurements provided by $\mathrm{ZGV}$ modes are attractive for investigating the bonding in a tri-layer structure without any contact. Using resonance frequencies of ZGV Lamb modes, Cho et al. reported on the evaluation of well- and weak-bonded adhesive plate samples. ${ }^{14,15}$ They observed a correlation between the first ZGV frequency and the bonding quality measured by mechanical tests.

\footnotetext{
a)Electronic mail: claire.prada@espci.fr
}

In this article, we investigate the evolution of the main $\mathrm{ZGV}$ modes in a tri-layer structure as a function of the longitudinal and shear interfacial stiffnesses. In Sec. II, the dispersion equation of a symmetrical tri-layered structure with interfacial stiffnesses is recalled and ZGV modes are discussed. Section III proposes a numerical study of the dependence of ZGV modes on the bonding layer thickness and on the interfacial stiffnesses. Experimental results obtained with a laser ultrasonic setup and a comparison with theoretical expectations are presented in Sec. IV.

\section{DISPERSION EQUATIONS AND ZGV MODES OF A TRI-LAYER}

The objective of this section is to establish the dispersion equation of a tri-layered structure following the approach chosen by Vlasie and Rousseau. ${ }^{4}$ Each layer is assumed to be isotropic, homogeneous, and infinite, with mass density $\rho_{i}$, longitudinal and transversal velocities $V_{L i}$ and $V_{T i}$, where $i$ indicates the layer number $(i=1,2,3)$. The first and third layer thicknesses are denoted by $h_{1}$ and $h_{3}$, whereas the bonding layer thickness is denoted $d$. The coupling between two successive layers is modeled by longitudinal and transversal springs of stiffnesses $K_{L j}$ and $K_{T j}$, where $j=1,2$ indicates the interface (Fig. 1). Spring stiffnesses can evolve from 0 (for uncoupled plates) up to $10^{17} \mathrm{~N} / \mathrm{m}^{3}$. The upper limit corresponds to an estimation, in an atomic chain model, of the epoxy layer bulk stiffnesses per unit length $\left(\mathrm{N} / \mathrm{m}^{3}\right.$ or $\left.\mathrm{Pa} / \mathrm{m}\right) .{ }^{16}$

In order to establish the dispersion law between the angular frequency $\omega$ and the wave number $k$, the scalar potential $\phi$ and the vector potential $\psi$ are used. The problem is supposedly two-dimensional so that the vector potential reduces to a scalar. The tangential and normal displacements derive from these potentials as follows: 


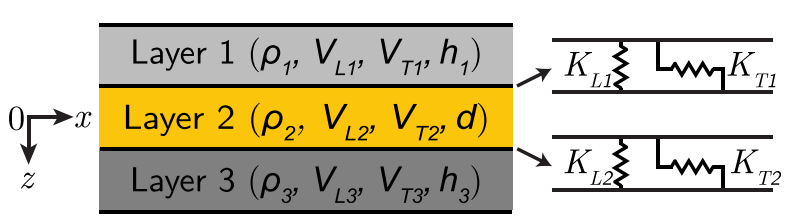

FIG. 1. (Color online) Geometry of the tri-layer model.

$$
u_{x}=\frac{\partial \phi}{\partial x}-\frac{\partial \psi}{\partial x}, \quad u_{z}=\frac{\partial \phi}{\partial x}+\frac{\partial \psi}{\partial x}
$$

and the stresses are given by:

$$
\begin{aligned}
& \sigma_{x z}=\mu\left(\frac{2 \partial^{2} \phi}{\partial x \partial z}+\frac{\partial^{2} \psi}{\partial x^{2}}-\frac{\partial^{2} \psi}{\partial z^{2}}\right), \\
& \sigma_{z z}=\lambda\left(\frac{\partial^{2} \phi}{\partial x^{2}}+\frac{\partial^{2} \phi}{\partial z^{2}}\right)+2 \mu\left(\frac{\partial^{2} \phi}{\partial z^{2}}+\frac{\partial^{2} \psi}{\partial x \partial z}\right),
\end{aligned}
$$

where $\lambda$ and $\mu$ are the Lamé coefficients. ${ }^{17}$ The potentials in the three layers are written as:

$$
\left\{\begin{array}{l}
\phi_{1}=\left(A_{1 L} \cos \left[p_{1}\left(z+\frac{d}{2}\right)\right]+B_{1 L} \sin \left[p_{1}\left(z+\frac{d}{2}\right)\right]\right) e^{\jmath(k x-\omega t)}, \\
\psi_{1}=\left(A_{1 T} \cos \left[q_{1}\left(z+\frac{d}{2}\right)\right]+B_{1 T} \sin \left[q_{1}\left(z+\frac{d}{2}\right)\right]\right) e^{\jmath(k x-\omega t)}, \\
\phi_{2}=\left(A_{2 L} \cos \left[p_{2} z\right]+B_{2 L} \sin \left[p_{2} z\right]\right) e^{\jmath(k x-\omega t)} \\
\psi_{2}=\left(A_{2 T} \cos \left[q_{2} z\right]+B_{2 T} \sin \left[q_{2} z\right]\right) e^{\jmath(k x-\omega t)} \\
\phi_{3}=\left(A_{3 L} \cos \left[p_{3}\left(z-\frac{d}{2}\right)\right]+B_{3 L} \sin \left[p_{3}\left(z-\frac{d}{2}\right)\right]\right) e^{\jmath(k x-\omega t)}, \\
\psi_{3}=\left(A_{3 T} \cos \left[q_{4}\left(z-\frac{d}{2}\right)\right]+B_{3 T} \sin \left[q_{3}\left(z-\frac{d}{2}\right)\right]\right) e^{\jmath(k x-\omega t)},
\end{array}\right.
$$

where $p$ and $q$ are the $z$-component of the longitudinal and transversal wave vectors, respectively. The wave numbers $k_{L i}=\omega / V_{L i}$ and $k_{T i}=\omega / V_{T i}$ satisfy dispersion equations of bulk waves $k_{L i}{ }^{2}=k^{2}+p_{i}{ }^{2}$ and $k_{T i}{ }^{2}=k^{2}+q_{i}{ }^{2} \cdot A_{i L}$ and $B_{i L}$ $\left(A_{i T}\right.$ and $\left.B_{i T}\right)$ are amplitudes of longitudinal (shear) components. At the free boundary, tangential $\left(\sigma_{x z}\right)$ and normal $\left(\sigma_{z z}\right)$ stresses vanish,

$$
\begin{cases}\sigma_{z z 1}=\sigma_{x z 1}=0 & \text { for } z=-h_{1}-d / 2 \\ \sigma_{z z 3}=\sigma_{x z 3}=0 & \text { for } z=h_{3}+d / 2\end{cases}
$$

The spring boundary conditions at both epoxy interfaces ${ }^{18}$ are written as follows:

$$
\begin{cases}\sigma_{z z 1}=\sigma_{z z 2}=K_{L 1}\left(u_{z 2}-u_{z 1}\right) & \text { for } z=-d / 2 \\ \sigma_{x z 1}=\sigma_{x z 2}=K_{T 1}\left(u_{x 2}-u_{x 1}\right) & \text { for } z=-d / 2 \\ \sigma_{z z 2}=\sigma_{z z 3}=K_{L 2}\left(u_{z 3}-u_{z 2}\right) & \text { for } z=d / 2 \\ \sigma_{x z 2}=\sigma_{x z 3}=K_{T 2}\left(u_{x 3}-u_{x 2}\right) & \text { for } z=d / 2\end{cases}
$$

Assuming a symmetric structure, the first and third layer properties, as well as the two longitudinal and normal springs, are identical and are denoted as $h, \rho_{1}, V_{L 1}, V_{T 1}, K_{L}$, and $K_{T}$, respectively. Then, it can be demonstrated that the system can be split into two sub-matrices, corresponding to symmetrical and anti-symmetrical solutions. Symmetrical modes fulfill the conditions

$$
\left\{\begin{array}{l}
u_{x}(z)=u_{x}(-z) \\
u_{z}(z)=-u_{z}(-z)
\end{array}\right.
$$

whereas anti-symmetrical modes satisfy

$$
\left\{\begin{array}{l}
u_{x}(z)=-u_{x}(z) \\
u_{z}(z)=u_{z}(-z)
\end{array}\right.
$$

Combining Eqs. (1), (4), (7), and (8) leads to: $A_{1 L}=A_{3 L}$, $B_{1 T}=B_{3 T}, A_{1 T}=-A_{3 T}, B_{1 L}=-B_{3 L}$, and $A_{2 T}=B_{2 L}=0$ for symmetrical modes and to: $A_{1 L}=-A_{3 L}, B_{1 T}=-B_{3 T}$, $A_{1 T}=A_{3 T}, B_{1 L}=B_{3 L}$, and $A_{2 L}=B_{2 T}=0$ for anti-symmetrical modes. Introducing the matrix

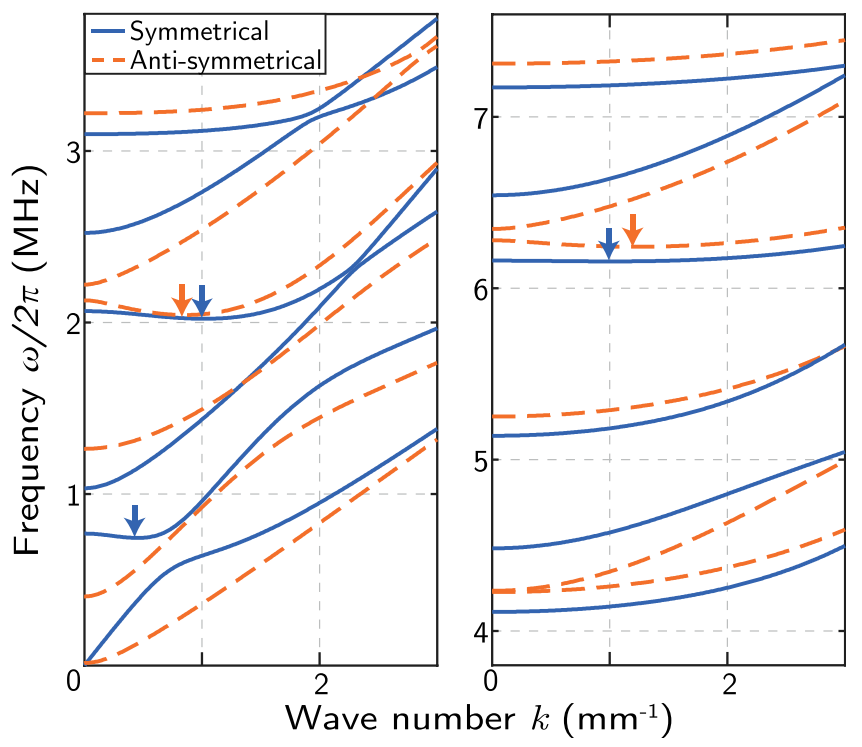

FIG. 2. (Color online) Theoretical dispersion curves for a Duralumin/epoxy/ Duralumin sample, with $K_{L}=K_{T}=5 \times 10^{14} \mathrm{~N} / \mathrm{m}^{3}$ and $h=50 \mu \mathrm{m}$. Arrows indicate ZGV Lamb mode frequencies. 


$$
M(\alpha)=\left[\begin{array}{cc}
2 \jmath k p_{1} \sin \left[p_{1} h\right] & 2 \jmath k p_{1} \cos \left[p_{1} h\right] \\
-\left(k_{t 1}^{2}-2 k^{2}\right) \cos \left[p_{1} h\right] & \left(k_{t 1}^{2}-2 k^{2}\right) \sin \left[p_{1} h\right] \\
\jmath k K_{T} & 2 \jmath k p_{1} \mu_{1} \\
-\left(k_{t 1}^{2}-2 k^{2}\right) \mu_{1} & p_{1} K_{L} \\
\jmath k K_{T} & 0 \\
0 & p_{1} K_{L}
\end{array}\right.
$$

$$
\begin{gathered}
0 \\
0 \\
-\jmath k K_{T} \cos \left[p_{2} \frac{d}{2}+\alpha\right] \\
-p_{2} K_{L} \sin \left[p_{2} \frac{d}{2}+\alpha\right] \\
2 \jmath k p_{2} \mu_{2} \sin \left[p_{2} \frac{d}{2}+\alpha\right]-\jmath k K_{T} \cos \left[p_{2} \frac{d}{2}+\alpha\right] \\
-\left(k_{t 2}^{2}-2 k^{2}\right) \mu_{2} \cos \left[p_{2} \frac{d}{2}+\alpha\right]-p_{2} K_{L} \sin \left[p_{2} \frac{d}{2}+\alpha\right]
\end{gathered}
$$

the boundary conditions can be written in matrix form by setting $\alpha=0$ for symmetrical modes and $\alpha=\pi / 2$ for antisymmetrical modes. It comes

$$
\begin{aligned}
& M_{S} U_{S}=0 \text { where } U_{S}= {\left[\begin{array}{l}
A_{1 L} \\
B_{1 L} \\
A_{1 T} \\
B_{1 T} \\
A_{2 L} \\
B_{2 T}
\end{array}\right] \text { and } M_{S}=M(\alpha=0), } \\
& M_{A} U_{A}=0 \text { where } U_{A}=\left[\begin{array}{l}
A_{1 L} \\
B_{1 L} \\
A_{1 T} \\
B_{1 T} \\
B_{2 L} \\
A_{2 T}
\end{array}\right] \text { and } M_{A}=M(\alpha=\pi / 2) .
\end{aligned}
$$

Non-trivial solutions are found when the determinant of matrices $M_{S}$ or $M_{A}$ vanishes. For symmetrical modes, $p_{1}, q_{1}$, and $q_{2}$ can be factorized in the second, fourth, and sixth rows, respectively, in matrix $M_{S}$. Similarly, $p_{1}, q_{1}$, and $p_{2}$ can be factorized for the second, fourth, and fifth row in matrix $M_{A}$. These terms correspond to bulk waves propagating at velocities $V_{L i}\left(p_{i}=0\right)$ and $V_{T i}\left(q_{i}=0\right)$. Such factorization avoids the unwanted bulk modes in the dispersion curves.

A numerical program was developed to calculate the dispersion curves of any isotropic tri-layer structures. Zeros of the secular equations are determined and the different modes are then distinguished. Once a mode is obtained, its phase velocity $\left(V_{\phi}=\omega / k\right)$ and its group velocity $\left(V_{g}=\partial \omega / \partial k\right)$ are calculated. A solution $(\omega, k)$ is a $\mathrm{ZGV}$ mode if $V_{g}=0$ with $k \neq 0$. Dispersion curves and ZGV Lamb waves are then

$$
\begin{array}{cc}
\left(k_{t 1}{ }^{2}-2 k^{2}\right) \cos \left[q_{1} h\right] & -\left(k_{t 1}{ }^{2}-2 k^{2}\right) \sin \left[q_{1} h\right] \\
2 \jmath k q_{1} \sin \left[q_{1} h\right] & 2 \jmath k q_{1} \cos \left[q_{1} h\right] \\
\left(k_{t 1}{ }^{2}-2 k^{2}\right) \mu_{1} & -q_{1} K_{T} \\
\jmath k K_{L} & 2 \jmath \mu_{1} k q_{1} \\
0 & -q_{1} K_{T} \\
\jmath k K_{L} & 0
\end{array}
$$

$$
\left.\begin{array}{c}
0 \\
0 \\
q_{2} K_{T} \cos \left[q_{2} \frac{d}{2}-\alpha\right] \\
\jmath k K_{L} \sin \left[q_{2} \frac{d}{2}-\alpha\right] \\
\left.-\left(k_{t 2}^{2}-2 k^{2}\right) \mu_{2} \sin \left[q_{2} \frac{d}{2}-\alpha\right]\right]+q_{2} K_{T} \cos \left[q_{2} \frac{d}{2}-\alpha\right] \\
2 \jmath k q_{2} \mu_{2} \cos \left[q_{2} \frac{d}{2}-\alpha\right]+\jmath k K_{L} \sin \left[q_{2} \frac{d}{2}-\alpha\right]
\end{array}\right],
$$

obtained for the tri-layer model using interfacial stiffnesses. Furthermore, once the dispersion curves are calculated, normal and tangential displacements $\left(u_{z}\right.$ and $u_{x}$, respectively) can also be obtained. For a given $(\omega, k)$ belonging to one branch of the dispersion curves, by setting one component common to $U_{S}$ and $U_{A}$ (e.g., $A_{1 L}=1$ ), the other components are determined from the boundary equation $[M][U]=[0]$. In the general case, i.e., if layers 1 and 3 are not identical, the resolution of Eqs. (4) and (5) leads to a $12 \times 12$ matrix which cannot be separated into two submatrices. Symmetrical and antisymmetrical modes no longer exist. In the presented example, the symmetrical tri-layer is composed of two Duralumin plates $\left(\rho_{1}=2795 \mathrm{~kg} / \mathrm{m}^{3}, V_{L 1}=6398 \mathrm{~m} / \mathrm{s}, V_{T 1}=3122 \mathrm{~m} / \mathrm{s}\right.$, $h=1.5 \mathrm{~mm})$ bonded with an epoxy layer $\left(\rho_{2}=1170 \mathrm{~kg} / \mathrm{m}^{3}\right.$, $\left.V_{L 2}=2500 \mathrm{~m} / \mathrm{s}, V_{T 2}=1100 \mathrm{~m} / \mathrm{s}, d=50 \mu \mathrm{m}\right)$. The chosen parameters correspond to measured values.

Figure 2 displays theoretical dispersion curves derived from Eq. (9) for values $K_{L}=K_{T}=5 \times 10^{14} \mathrm{~N} / \mathrm{m}^{3}$ corresponding to a relatively weak coupling. The first three branches correspond to $A_{0}, S_{0}$, and $A_{1}$ Lamb modes and like in a single plate do not present any ZGV mode. Five ZGV modes are visible on higher order branches. They can be ascribed to the repulsion of two modes. ${ }^{19}$ These interactions only occur for modes of the same symmetry having close enough cutoff frequencies.

The number of ZGV Lamb modes can then vary with the different parameters. This phenomenon is discussed in Sec. III [Figs. 4, 5(a), and 6]. The first ZGV Lamb mode at frequency $f=0.744 \mathrm{MHz}$ only exists in a tri-layer plate. According to the displacement distribution, this mode corresponds to the $S_{1} S_{2} \mathrm{ZGV}$ mode of the whole structure and is denoted as so hereafter [Fig. 3(a)]. Around 2, 6, and $10 \mathrm{MHz}, \mathrm{ZGV}$ Lamb modes can be associated in pairs (see Fig. 2 for the pairs around 2 and $6 \mathrm{MHz}$ ). These pairs correspond to symmetrical and anti-symmetrical coupling of the 


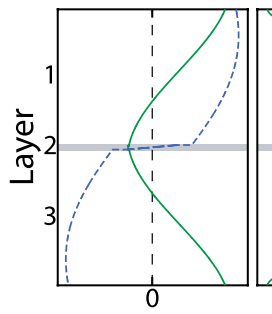

(a)

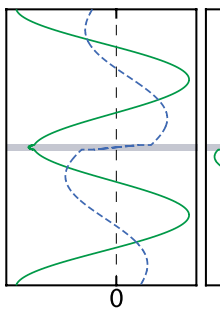

(b)

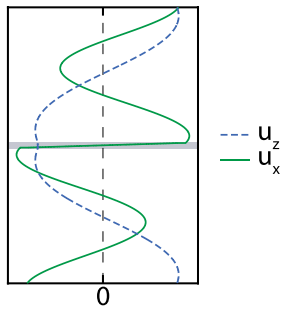

(c)

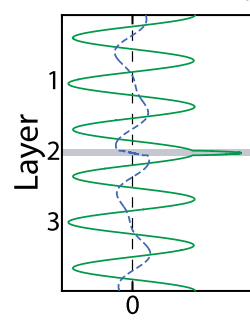

(d)

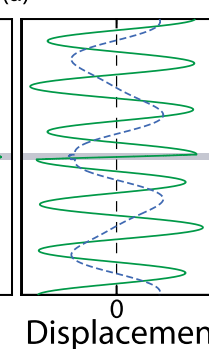

(e)

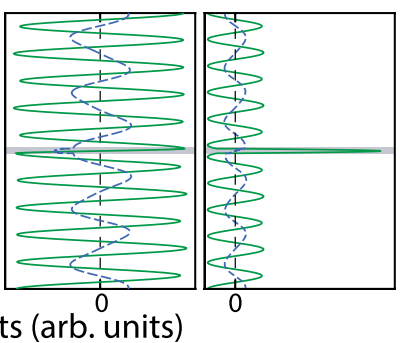

(g)
FIG. 3. (Color online) Theoretical normal (dashed line) and in-plane (solid line) displacements, in a Duralumin/epoxy/Duralumin structure with $K_{L}=K_{T}$ $=5 \times 10^{14} \mathrm{~N} / \mathrm{m}^{3}$ and $h=50 \mu \mathrm{m}$, for ZGV Lamb modes (a) $S_{1} S_{2}(0.744 \mathrm{MHz})$, (b) $S\left(S_{1} S_{2}\right)(2.022 \mathrm{MHz}),\left(\right.$ c) $A\left(S_{1} S_{2}\right)(2.032 \mathrm{MHz})$, (d) $S\left(S_{3} S_{6}\right)^{*}(6.173 \mathrm{MHz})$, (e) $A\left(S_{3} S_{6}\right)^{*}(6.222 \mathrm{MHz})$, (f) $A\left(S_{5} S_{10}\right)^{*}(10.403 \mathrm{MHz})$, and (g) $S\left(S_{5} S_{10}\right)^{*}$ (10.685 MHz).

same ZGV modes of the single plate. Figure 3 displays the normal and shear displacements of the first seven $\mathrm{ZGV}$ Lamb modes for the same intermediate bonding $\left(K_{L}=K_{T}=5 \times 10^{14} \mathrm{~N} / \mathrm{m}^{3}\right)$.

From Figs. 3(b) and 3(c), it appears that the two modes around $2 \mathrm{MHz}$ correspond to the ZGV Lamb mode $S_{1} S_{2}$ in a single Duralumin plate where both plates are coupled by the intermediate layer symmetrically and anti-symmetrically, respectively. Their frequencies remain close: 2.022 and 2.032 MHz to be compared with $1.915 \mathrm{MHz}$ for a single 1.5$\mathrm{mm}$ thick Duralumin plate. The corresponding modes are denoted $S\left(S_{1} S_{2}\right)$ and $A\left(S_{1} S_{2}\right)$ hereafter. A similar observation is possible for higher frequency modes display in Fig. 3: ZGV Lamb modes around $6 \mathrm{MHz}$ [Figs. 3(d) and 3(e)] and $10 \mathrm{MHz}$
[Figs. 3(f) and 3(g)] are associated to $S_{3} S_{6}(f=6.219 \mathrm{MHz})$ and $S_{5} S_{10}(f=10.406 \mathrm{MHz})$ in a single Duralumin plate, and are denoted $S\left(S_{3} S_{6}\right)^{*}, A\left(S_{3} S_{6}\right)^{*}, A\left(S_{5} S_{10}\right)^{*}$, and $S\left(S_{5} S_{10}\right)^{*}$ in the following. The asterisk index indicates that the two modes interacting to create the repulsion differ from the two interacting for lower bonding (see Sec. III A) but the displacements are very similar in both cases.

\section{PARAMETRIC STUDY}

In order to understand the behavior of the $\mathrm{ZGV}$ mode frequencies, a parametric study is conducted for the same symmetric tri-layer. The Duralumin plate thicknesses and the elastic parameters of all layers are maintained constant, while the epoxy layer thickness and the interfacial stiffnesses are varied successively.

\section{A. Influence of the bonding layer thickness}

The influence of the layer thickness is studied for a strong coupling $\left[K_{L}=K_{T}=10^{17} \mathrm{~N} / \mathrm{m}^{3}\right.$, Fig. 4(a) $]$ and for a weak coupling $\left[K_{L}=K_{T}=10^{14} \mathrm{~N} / \mathrm{m}^{3}\right.$, Fig. 4(b)]. Several $\mathrm{ZGV}$ modes appear in both cases. Their existence is limited to a thickness range, except for the $\mathrm{ZGV}$ mode associated to the lowest frequency $\left(f_{S_{1} S_{2}}\right)$, that exists for all thicknesses. For a layer thickness above $\sim 20 \mu \mathrm{m}$, modes $S\left(S_{1} S_{2}\right)$ and $A\left(S_{1} S_{2}\right)$ are present at frequencies around $2 \mathrm{MHz}$. Modes $S\left(S_{3} S_{6}\right)$ and $A\left(S_{3} S_{6}\right)$ around $6 \mathrm{MHz}$ and modes $S\left(S_{5} S_{10}\right)$ and $A\left(S_{5} S_{10}\right)$ around $10 \mathrm{MHz}$ are also present although for some values of $d$, one or two of these modes is absent. In Figs. 4(a) and 4(b), around 6 and $10 \mathrm{MHz}$, a ZGV branch is disappearing and another branch is then appearing at a close frequency. Simulations show that displacements associated to those ZGV branches are similar. Thus, the same notation is used for these modes and an asterisk indicates the branch change. It could be noted that for a very thin intermediate layer $(h \leq 10 \mu \mathrm{m})$ and a strong bonding, four other modes appear [Fig. 4(a)]; they are not observed for lower spring stiffnesses [Fig. 4(b)]. These modes correspond to ZGV

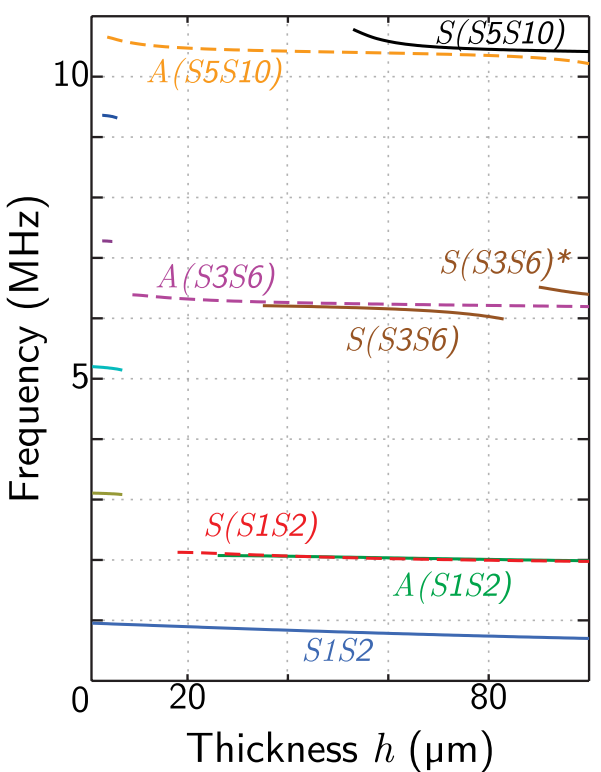

(a)

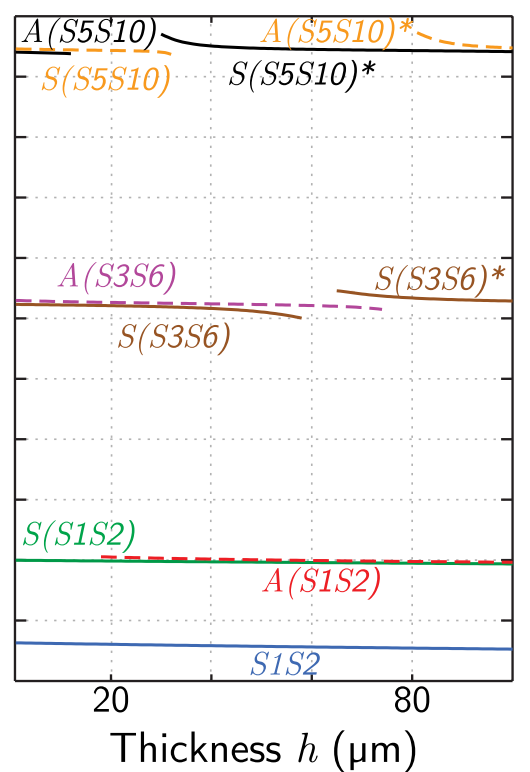

(b)
FIG. 4. (Color online) Theoretical $\mathrm{ZGV}$ Lamb mode frequencies as a function of the bonding layer thickness $d$, for a Duralumin/epoxy/Duralumin tri-layer, with (a) $K_{L}=K_{T}=10^{17} \mathrm{~N} /$ $\mathrm{m}^{3}$ and (b) $K_{L}=K_{T}=10^{14} \mathrm{~N} / \mathrm{m}^{3}$. Symmetrical modes are in solid lines whereas anti-symmetrical modes are in dashed lines. 


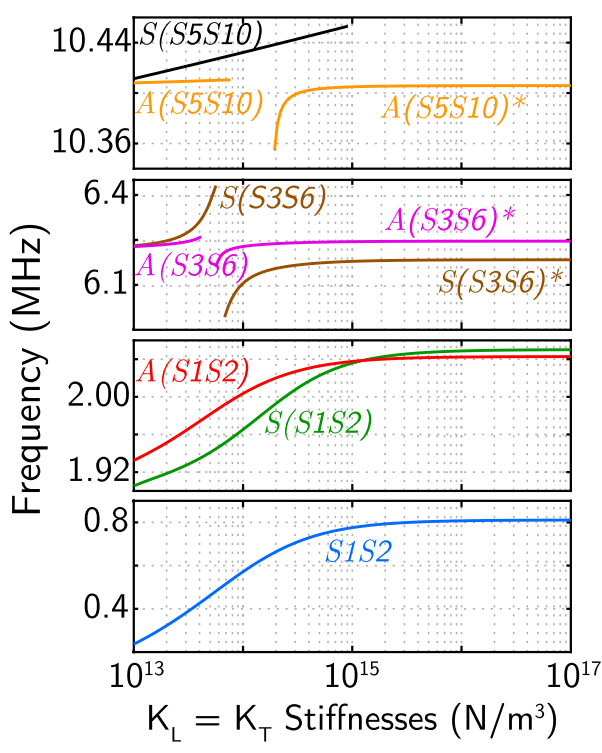

(a)

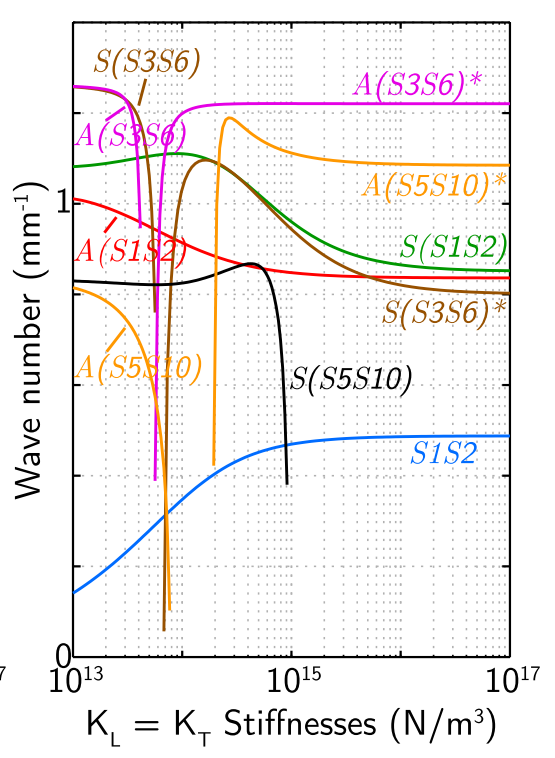

(b)
FIG. 5. (Color online) Theoretical ZGV mode frequencies (a) and associated wave numbers (b), for $d=50 \mu \mathrm{m}$, as a function of stiffnesses $K_{L}=K_{T}$ varying from $10^{13}$ to $10^{17} \mathrm{~N} / \mathrm{m}^{3}$. Symmetrical modes (solid line) and anti-symmetrical modes (dashed line). modes in a single plate having twice the thickness of the duralumin plates. For example, frequencies and displacements of the first two additional modes correspond to $S_{3} S_{6}$ and $S_{5} S_{10} \mathrm{ZGV}$ modes of a $3 \mathrm{~mm}$ thick duralumin plate, respectively.

As expected, all ZGV frequencies decrease with increasing bonding layer thickness. However, frequency variations differ from one mode to the other. The ZGV frequency $f_{S_{1} S_{2}}$ decays by $26.5 \%$ for $K_{L}=K_{T}=10^{17} \mathrm{~N} / \mathrm{m}^{3}$ [Fig. 4(a)]. This highlights the necessity of an accurate knowledge of the value of each layer thickness before considering the interfacial stiffnesses. Other layer properties involved in the $\mathrm{ZGV}$ calculation $\left(\rho_{2}, V_{L 2}, V_{T 2}\right)$ also influence the $\mathrm{ZGV}$ Lamb mode frequencies. However, such properties can be measured before the bonding, and are assumed to be homogeneous. On the contrary, the glue layer thickness can locally evolve and simulations demonstrate the need to precisely determine the layer thickness.

\section{B. Influence of longitudinal and shear stiffnesses}

For a bi-layer structure, it was shown that symmetrical modes only vary with the longitudinal interfacial stiffness, while anti-symmetrical ones only vary with the shear interfacial stiffness. ${ }^{11}$ The situation is more complex for a tri-layer structure where symmetric and anti-symmetric modes depend on both $K_{L}$ and $K_{T}$ [Eq. (9)]. To understand the dependence of resonance frequencies with interfacial stiffnesses, we first set the bonding layer thickness to $50 \mu \mathrm{m}$ and assume $K_{L}=K_{T}$. Figure 5 displays the evolution of $\mathrm{ZGV}$ frequencies for symmetrical and anti-symmetrical modes, as a function of the stiffnesses varying over 4 decades. It appears that the frequencies of all $\mathrm{ZGV}$ modes increase with increasing stiffnesses and that the lowest $\mathrm{ZGV}$ mode $S_{1} S_{2}$ is, again, always present. As shown for a bi-layer structure, this low frequency mode characterizes the presence of a longitudinal coupling between the two plates. ${ }^{11}$ The ZGV frequency $f_{S_{1} S_{2}}$ exists for any $K_{L}$ and $K_{T}$ positive values. Its normal component of the displacement is significant [Fig. 3(a)] for any $K_{L}, K_{T}$ values, which allows its optical detection (Sec. IV). ZGV resonances associated to modes in the intermediate layer also exist but their frequencies are much higher because of the intermediate layer thickness $(d=50 \mu \mathrm{m})$ : the first one appears around $22 \mathrm{MHz}$. As the mechanical displacement is mainly localized in the bonding layer, these ZGV modes are not experimentally detected and then not discussed.

The wavelength of ZGV modes should also be considered to optimize the excitation spot size. For a homogeneous plate, the ratio of the wavelength to the plate thickness depends only on the Poisson ratio, ${ }^{20}$ but in a three-layer structure, it depends on several parameters. For example, Fig. 5(b) shows that the wavelength depends on the ZGV mode order and either increases or decreases with the spring stiffnesses $K_{L, T}$. The beginning of a ZGV branch is associated to a low wave number which increases with $K_{L, T}$ [e.g., $A\left(S_{3} S_{6}\right)^{*}$ in Fig. 5(b)]. Similarly, the wave number vanishes at the end of a ZGV branch [e.g., $A\left(S_{5} S_{10}\right)$ in Fig. 5(b)]. The $S_{1} S_{2} \mathrm{ZGV}$ mode wave number is close to zero for very low stiffnesses and increases with $K_{L, T}$. This behavior can be ascribed to the existence of the $S_{1} S_{2}$ mode for any strictly positive spring stiffnesses. At the opposite, the existence of other modes in a single plate (i.e., for $K_{L}=K_{T}=0$ ) explains their non-zero wave number for low spring stiffnesses.

Figure 6 presents the evolution of the first five $\mathrm{ZGV}$ mode frequencies as a function of $K_{T}$ with $K_{L}=10^{14} \mathrm{~N} / \mathrm{m}^{3}$ (a) and of $K_{L}$ with $K_{T}=10^{14} \mathrm{~N} / \mathrm{m}^{3}$ (b).

For a bi-layer structure, it was demonstrated that symmetrical (anti-symmetrical) ZGV modes only depends on $K_{L}$ $\left(K_{T}\right) .{ }^{11}$ For a tri-layer structure, this is no longer true. However, in our experiment the bonding layer thickness is small compared to the plate one and similar behaviors are observed. Therefore, each ZGV mode is mostly influenced by one or the other interfacial stiffness. In particular $f_{S_{1} S_{2}}$ and $f_{S\left(S_{1} S_{2}\right)}$ mainly depend on $K_{L}$ [Fig. 6(b)], whereas $f_{A\left(S_{1} S_{2}\right)}$ primarily depends on $K_{T}$ [Fig. 6(a)]. These observations will 


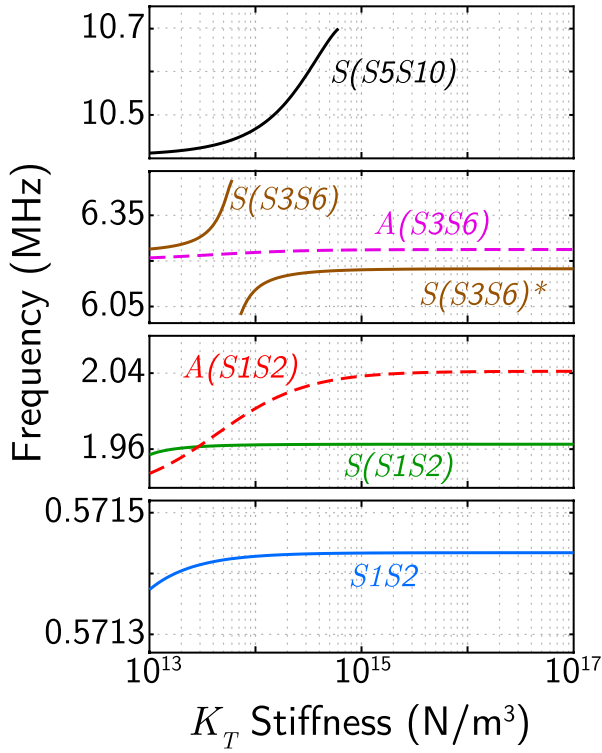

(a)

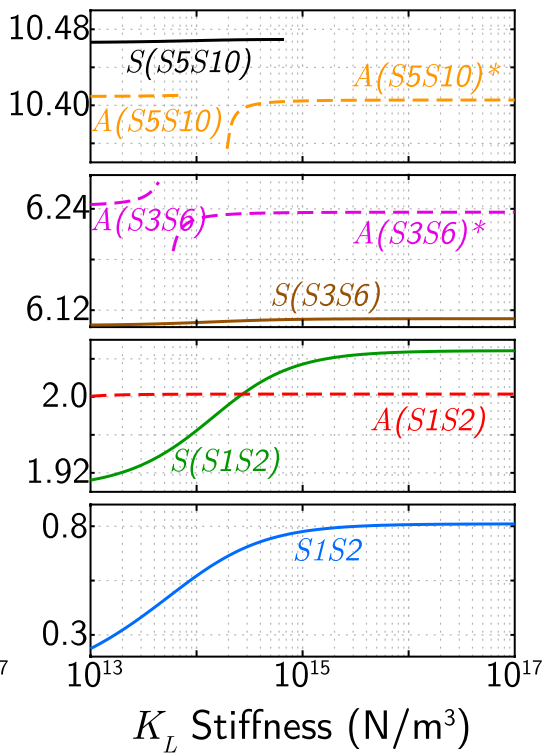

(b) guide the experimental procedure to determine the interfacial stiffnesses.

\section{EXPERIMENTS}

An all-optical setup is used to measure $\mathrm{ZGV}$ resonances. Lamb waves are excited by a Q-switched Nd:YAG (yttrium aluminum garnet) laser (Quantel Laser, France, Centurion, $1064 \mathrm{~nm}$ ) which delivers 10-ns pulses of 8-mJ energy. The normal surface displacement is detected on the opposite side of the plate with an interferometer (Bossa Nova Tech., Venice, CA, Tempo1D, $532 \mathrm{~nm}$ ). The structure is a $100 \times 100 \mathrm{~mm}^{2}$ tri-layer composed of two Duralumin plates of thickness $h=1.50 \mathrm{~mm}$ bonded by a bi-component epoxy adhesive (Loctite Hysol, France, 9466 A\&B). Substrate surfaces were cleaned with acetone. To control the adhesive joint thickness, thin metallic spacers are disposed along the edges prior to then spreading of the glue. The specimen was cured at room temperature $\left(22{ }^{\circ} \mathrm{C}\right)$ and then maintained under a $5-\mathrm{kN}$ constant pressure during $12 \mathrm{~h}$ with an automatic mechanical testing machine (MTS, Eden Prairie, MN QTest/ 25). The thickness spacers were $43-\mu \mathrm{m}$ thick. However, in the middle of the plate, where the experiment is achieved, the layer thickness is smaller due to the hardening process of the glue. The determined mass densities are $\rho_{1}=2795 \mathrm{~kg} / \mathrm{m}^{3}$ for Duralumin and $\rho_{2}=1170 \mathrm{~kg} / \mathrm{m}^{3}$ for the epoxy. The longitudinal and shear velocities were measured on a single Duralumin plate using ZGV Lamb modes: ${ }^{19} V_{L 1}=6398 \mathrm{~m} / \mathrm{s}$ and $V_{T 1}=3122 \mathrm{~m} / \mathrm{s}$. The longitudinal and shear acoustic wave velocities of epoxy are estimated using piezoelectric contact transducers (Olympus, USA, V103 and V153) $V_{L 2}=2500 \mathrm{~m} / \mathrm{s}$ and $V_{T 2}=1100 \mathrm{~m} / \mathrm{s}$.

For an accurate knowledge of the bonding layer thickness, the precursors in the transmitted signal are obtained with a small laser beam diameter $(\sim 100 \mu \mathrm{m}$ at $1 / \mathrm{e})$ [Fig. $7(\mathrm{a})$. The first arrival, at $t_{0}$, corresponds to the direct transmission in the structure while the second arrival, at $t_{1}$, is associated with the wave twice reflected in the intermediate layer. The measured times $t_{0}=601.6 \pm 0.4 \mathrm{~ns}$ and $t_{1}=629.6 \pm 0.4 \mathrm{~ns}$ lead to a glue layer thickness $d=35 \pm 1$ $\mu \mathrm{m}$.

The local resonance spectrum is measured at the same point with an enlarged spot. A $\sim 3-\mathrm{mm}$ beam diameter (at $1 / e$ ) is chosen as a good compromise to excite the first $\mathrm{ZGV}$ modes [Fig. 5(b) and Balogun et al. ${ }^{20}$ ]. The resulting signal and its Fourier transform are displayed in Figs. 7(b) and Fig. 8, respectively. Several ZGV resonances are observed with a precision estimated to be $1 \mathrm{kHz}$ [see Table I]. The first one at $f=0.836 \mathrm{MHz}$ corresponds to the $S_{1} S_{2}$ mode. The following two around $2.1 \mathrm{MHz}$ correspond to $S\left(S_{1} S_{2}\right)$ and $A\left(S_{1} S_{2}\right)$, not yet discriminated. Similarly, two resonances around $6.2 \mathrm{MHz}$ can be attributed to $S\left(S_{3} S_{6}\right)$ and $A\left(S_{3} S_{6}\right)$. Around $10 \mathrm{MHz}$, only one resonance is detected. This is probably due to the fact that the displacement associated to the $S\left(S_{5} S_{10}\right) \mathrm{ZGV}$ mode is mainly localized in the epoxy layer and not at the free surface [see Fig. 3(g)].

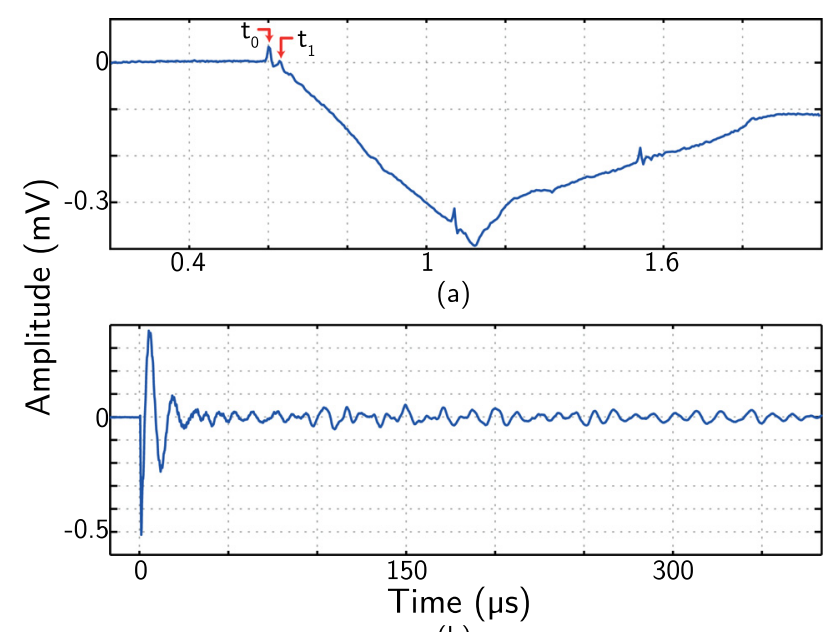

(b)

FIG. 7. (Color online) Typical signal measured by transmission in the Duralumin/epoxy/Duralumin structure: (a) at short arrival with a $\sim 100 \mu \mathrm{m}$ beam diameter to observe first reflections in the intermediate layer, (b) with a beam diameter of $\sim 3 \mathrm{~mm}$ to enhance $\mathrm{ZGV}$ mode resonances. 




FIG. 8. (Color online) Experimental signal obtained by transmission in the Duralumin/epoxy/Duralumin tri-layer structure.

In order to determine the interfacial stiffnesses, the frequency $f_{S_{1} S_{2}}$ and $f_{A\left(S_{1} S_{2}\right)}$ are calculated as a function of both spring stiffnesses in the range $\left[10^{13} ; 10^{17}\right] \mathrm{N} / \mathrm{m}^{3}$. The resulting maps are displayed in Figs. 9(a) and 9(b). From the isofrequency curves, it clearly appears that $f_{S_{1} S_{2}}$ mostly depends on $K_{L}$ while $f_{A\left(S_{1} S_{2}\right)}$ mostly depends on $K_{T}$. In the experiment, it is necessary to figure out which one of the two resonances measured around $2 \mathrm{MHz}$ corresponds to the anti-symmetrical mode $A\left(S_{1} S_{2}\right)$.

The discrimination can be done from the resonance width. As shown in Fig. 3(c) for the anti-symmetrical mode, the glue layer is mainly sheared, leading to a stronger attenuation than for the symmetrical ones, where the layer is weakly sheared [Fig. 3(b)]. Consequently, the broader peak at $2.055 \mathrm{MHz}$ can be ascribed to the anti-symmetrical mode $A\left(S_{1} S_{2}\right)$, and the thinner one at $2.066 \mathrm{MHz}$ to the $S\left(S_{1} S_{2}\right)$ mode [Fig. 8]. A similar observation can be done for $S\left(S_{3} S_{6}\right)$ at $6.215 \mathrm{MHz}$ and $A\left(S_{3} S_{6}\right)$ at $6.247 \mathrm{MHz}$. Moreover, if we consider the reverse case where $A\left(S_{1} S_{2}\right)$ resonance would be at $f=2.066 \mathrm{MHz}$, the obtained set of $K_{L}$ and $K_{T}$ values increases the discrepancies between theoretical and experimental $\mathrm{ZGV}$ frequencies. This confirms the identification of the symmetrical and antisymmetrical ZGV modes. Consequently, the isofrequency curves are plotted at $f=2.055 \mathrm{MHz}$ for the anti-symmetrical mode $A\left(S_{1} S_{2}\right)$ [Fig. 9(b)] and at $f=0.836 \mathrm{MHz}$ for the $S_{1} S_{2}$ mode [Fig. 9(a)].

The interfacial stiffnesses correspond to the intersection of these two curves and are found to be $K_{L}=2.52 \times 10^{15}$ and $K_{T}=2.83 \times 10^{14} \mathrm{~N} / \mathrm{m}^{3}$ for a thickness $d=35 \mu \mathrm{m}$. For these values, the theoretical frequencies of the other $\mathrm{ZGV}$ modes fit the measured peaks with a discrepancy below $0.3 \%$ [Table I]. For $d$ varying in the range 34 to $36 \mu \mathrm{m}$, which corresponds to the layer thickness uncertainty, $K_{L}$

TABLE I. Experimental and theoretical ZGV Lamb mode frequencies.

\begin{tabular}{lcc}
\hline \hline Experiment $(\mathrm{MHz})$ & Theory $(\mathrm{MHz})$ & Difference $(\mathrm{kHz})$ \\
\hline 0.836 & 0.836 & - \\
2.055 & 2.055 & - \\
2.066 & 2.063 & -3 \\
6.215 & 6.204 & -11 \\
6.247 & 6.260 & 13 \\
10.424 & 10.426 & 2 \\
\hline \hline
\end{tabular}

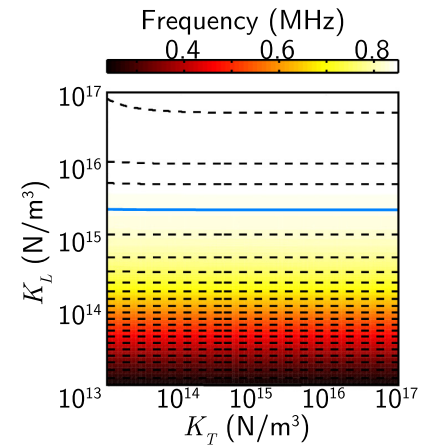

(a)

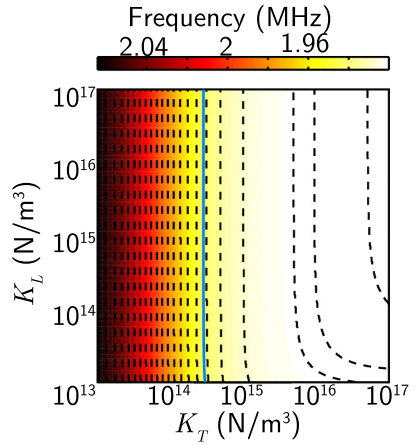

(b)
FIG. 9. (Color online) Theoretical evolution of the frequency of the modes $S_{1} S_{2}$ (a) and $A\left(S_{1} S_{2}\right)$ (b) as a function of both interfacial stiffnesses in the range $\left[10^{13} ; 10^{17}\right] \mathrm{N} / \mathrm{m}^{3}$. Dashed lines correspond to isofrequency curves. Solid lines correspond to $f_{S_{1} S_{2}}=0.836 \mathrm{MHz}$ (a) and $f_{A\left(S_{1} S_{2}\right)}=2.055 \mathrm{MHz}(\mathrm{b})$.

varies from 2.15 to $3.07 \times 10^{15} \mathrm{~N} / \mathrm{m}^{3}$ and $K_{T}$ varies from 2.56 to $3.17 \times 10^{14} \mathrm{~N} / \mathrm{m}^{3}$.

Similar values for the longitudinal stiffness are found in the literature for aluminum/epoxy interface. ${ }^{21,22}$ The ratio $K_{T} / K_{L}$ equal to 0.11 is much lower that the values of 0.7 to 0.8 reported by Cantrell. ${ }^{23}$ However this ratio is close to the value 0.16 measured by Guo et al. in the case of a film deposited on a steel substrate. ${ }^{24}$ It should be noted that parameters $K_{T}$ and $K_{L}$ depend on many factors during the hardening process of the bonding layer such as humidity or surface roughness.

Dispersion curves were measured by shifting the source (diameter spot $0.1 \mathrm{~mm}$ ) from the probe beam, along $40 \mathrm{~mm}$ by $0.1 \mathrm{~mm}$ steps, and applying a two-dimensional Fourier transform to the acquired signals. Experimental curves are displayed in Fig. 10. Theoretical dispersion curves were

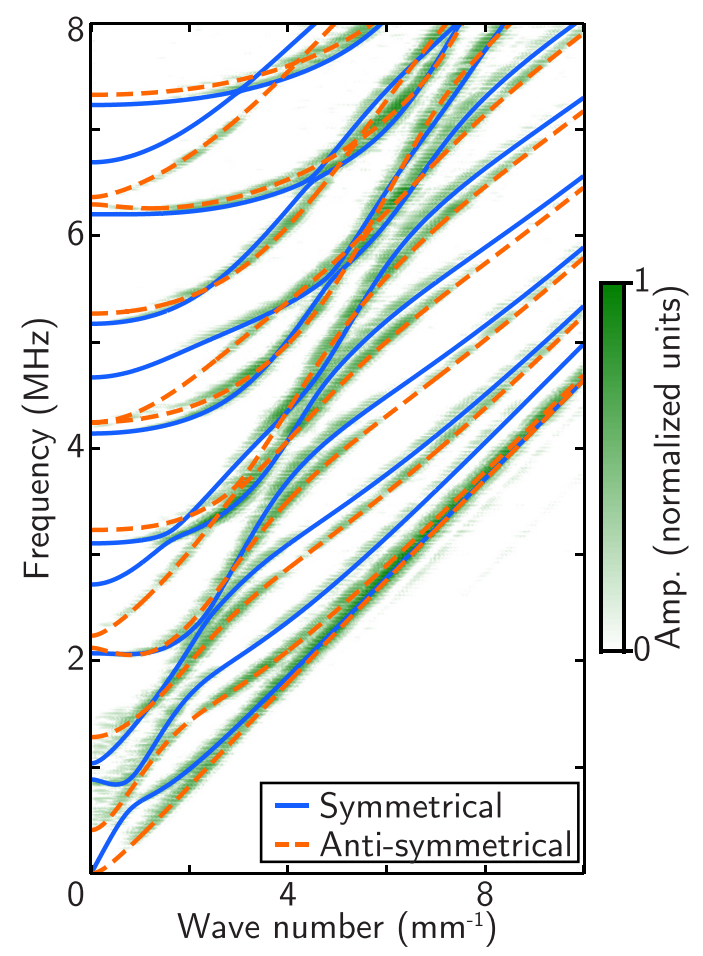

FIG. 10. (Color online) Experimental (map) and theoretical (solid and dashed lines) dispersion curves obtained with the measured stiffnesses $K_{L}=2.52 \times 10^{15} \mathrm{~N} / \mathrm{m}^{3}$ and $K_{T}=2.83 \times 10^{14} \mathrm{~N} / \mathrm{m}^{3}$. 
calculated with the stiffnesses previously obtained and then superposed to experimental data. A good agreement between theoretical and experimental curves is observed for a large range of $(\omega, k)$. It confirms that, provided the elastic properties and thickness of the epoxy layer are known, ZGV frequencies are sufficient to determine the local interfacial stiffnesses of the structure.

\section{CONCLUSION}

A method using ZGV Lamb modes is developed to characterize the bonding in a tri-layer structure. Theoretical and experimental studies are carried out in the symmetrical case of two Duralumin plates bonded by an epoxy layer. The coupling at both epoxy/metal interfaces is modeled by normal and tangential stiffnesses, $K_{L}$ and $K_{T}$. The parametric study demonstrates the sensitivity of ZGV Lamb modes to $K_{L, T}$ and to the bonding layer thickness. In general, the first symmetrical and anti-symmetrical ZGV Lamb modes are mostly sensitive to normal and shear stiffnesses, respectively. Provided the bonding layer thickness is well known, the interfacial stiffnesses can be evaluated from $\mathrm{ZGV}$ frequency measurements. To this end, the low frequency ZGV Lamb mode is used to estimate $K_{L}$, and then the second ZGV Lamb mode is used to estimate $K_{T}$. The difference between theoretical and the other experimental ZGV frequencies is found to be less than $0.3 \%$, which shows the potential interest of this technique.

In the future, measurements with different glue thicknesses or adhesive layers could be investigated to compare different bonding qualities. It would be insightful to couple $\mathrm{ZGV}$ interfacial stiffness measurements with mechanical tensile tests.

\section{ACKNOWLEDGMENTS}

This work was supported by LABEX WIFI (Laboratory of Excellence ANR-10-LABX-24) within the French Program "Investments for the Future" under reference ANR10-IDEX-0001-02 PSL* and by the Chaire SAFRAN ESPCI.

${ }^{1}$ S. Rokhlin, A. I. Lavrentyev, and B. Li, "Ultrasonic evaluation of environmental durability of adhesive joints," Res. Nondestr. Eval. 5, 95-109 (1993).

${ }^{2}$ M. K. Budzik, B. Mascaro, J. Jumel, M. Castaings, and M. E. R. Shanahan, "Monitoring of crosslinking of a DGEBA-PAMAM adhesive in composite/aluminium bonded joint using mechanical and ultra-sound techniques," Int. J. Adhes. Adhes. 35, 120-128 (2012).

${ }^{3}$ B. Le Crom and M. Castaings, "Shear horizontal guided wave modes to infer the shear stiffness of adhesive bond layers," J. Acoust. Soc. Am. 127, 2220-2230 (2010).
${ }^{4} \mathrm{~V}$. Vlasie and M. Rousseau, "Acoustical validation of the rheological models for a structural bond," Wave Motion 37, 333-349 (2003).

${ }^{5}$ V. Vlasie, S. de Barros, M. Rousseau, and L. Champaney, "Ultrasonic rheological model of cohesive and adhesive zones in aluminum joints: Validation by mechanical tests," Arch. Appl. Mech. 75, 220-234 (2006).

${ }^{6}$ N. Alaoui Ismaili, C. De Mello da Silva, M. Ech-Cherif El-Kettani, G. Despaux, M. Rousseau, and J.-L. Izbicki, "Lamb modes for the characterization of bonded structures," Acta. Acust. 99, 331-334 (2013).

${ }^{7}$ D. Cerniglia, A. Pantano, and N. Montinaro, "3D simulations and experiments of guided wave propagation in adhesively bonded multi-layered structures," NDT\&E Int. 43, 527-535 (2010).

${ }^{8} \mathrm{M}$. Castaings, "SH ultrasonic guided waves for the evaluation of interfacial adhesion," Ultrasonics 54, 1760-1775 (2014).

${ }^{9}$ K. Nishimiya, K. Yamamoto, K. Mizutani, and N. Wakatsuki, "Negative group velocities of Lamb-type waves in a glass/water/glass structure controlled by the thickness of water layer,” Jpn. J. Appl. Phys. 46, 4483-4485 (2007).

${ }^{10}$ K. Nishimiya, K. Mizutani, N. Wakatsuki, and K. Yamamoto, "Relationships between existence of negative group velocity and physical parameters of materials for Lamb-type waves in solid/liquid/solid structure,” Jpn. J. Appl. Phys. 47, 3855-3858 (2008).

${ }^{11}$ S. Mezil, J. Laurent, D. Royer, and C. Prada, "Non-contact probing of interfacial stiffnesses between two plates by zero-group velocity Lamb modes," Appl. Phys. Lett. 105, 021605 (2014).

${ }^{12}$ D. Clorennec, C. Prada, and D. Royer, "Laser ultrasonic inspection of plates using zero-group velocity Lamb modes," IEEE Trans. Ultrason. Ferroelectr. Freq. Control 57, 1125-1132 (2010).

${ }^{13}$ H. Cho, D. Oka, and T. Matsuo, "Damage evaluation of unsaturated polyester resin using zero-group velocity Lamb waves in non-contact matter," J. Solid Mech. Mater. Eng. 6, 678-684 (2012).

${ }^{14} \mathrm{H}$. Cho, Y. Hara, and T. Matsuo, "Evaluation of the thickness and bond quality of three-layered media using zero-group-velocity Lamb waves," J. Phys. Conf. Ser. 520, 012023 (2014).

${ }^{15} \mathrm{H}$. Cho, Y. Yaguchi, and H. Ito, "Characterization of the bond quality of adhesive plates utilizing zero-group-velocity Lamb waves measured by a laser ultrasonics technique," Mech. Eng. J. 2, 1 (2015).

${ }^{16}$ D. Royer and E. Dieulesaint, "Elastic waves," in Solids II: Generation, Acousto-Optic Interaction, Applications (Springer, New York, 2000).

${ }^{17}$ J. D. Achenbach, Wave Propagation in Elastic Solids (Elsevier, NorthHolland, Amsterdam, 1984).

${ }^{18}$ J. P. Jones and J. S. Whittier, "Waves at a flexibly bonded interface," J. Appl. Mech. 34, 905-909 (1967).

${ }^{19}$ D. Clorennec, C. Prada, and D. Royer, "Local and noncontact measurements of bulk acoustic wave velocities in thin isotropic plates and shells using zero group velocity Lamb modes," J. Appl. Phys. 101, 034908 (2007).

${ }^{20}$ O. Balogun, T. W. Murray, and C. Prada, "Simulation and measurement of the optical excitation of the $S_{1}$ zero group velocity Lamb wave resonance in plates," J. Appl. Phys. 102, 064914 (2007).

${ }^{21}$ V. V. Belloncle and M. Rousseau, "Ultrasonic measurements of pretreated aluminium joints durability during hot cycles," Res. Nondestruct. Eval. 20, 131-144 (2009).

${ }^{22}$ S.-C. Her and Y.-C. Lin, "Assessment of adhesive bond strength using the ultrasonic technique,” J. Adhes. 90, 545-554 (2014).

${ }^{23}$ J. H. Cantrell, "Determination of absolute bond strength from hydroxyl groups at oxidized aluminum-epoxy interfaces by angle beam ultrasonic spectroscopy,” J. Appl. Phys. 96, 3775-3781 (2004).

${ }^{24}$ Z. Guo, J. Achenbach, A. Madan, K. Martin, and M. Graham, "Modeling and acoustic microscopy measurements for evaluation of the adhesion between a film and a substrate," Thin Solid Films 394, 188-200 (2001). 